\title{
A remark on separate holomorphy
}

\author{
by \\ Marek Jarnicki (Kraków) and Peter Pflug (Oldenburg)
}

\begin{abstract}
Let $X$ be a Riemann domain over $\mathbb{C}^{k} \times \mathbb{C}^{l}$. If $X$ is a domain of holomorphy with respect to a family $\mathcal{F} \subset \mathcal{O}(X)$, then there exists a pluripolar set $P \subset \mathbb{C}^{k}$ such that every slice $X_{a}$ of $X$ with $a \notin P$ is a region of holomorphy with respect to the family $\left\{\left.f\right|_{X_{a}}: f \in \mathcal{F}\right\}$.
\end{abstract}

1. Introduction: Riemann regions of holomorphy. Let $(X, p)$ be a Riemann region over $\mathbb{C}^{n}$, i.e. $X$ is an $n$-dimensional complex manifold and $p: X \rightarrow \mathbb{C}^{n}$ is a locally biholomorphic mapping (see [Jar-Pfl 2000] for details). If $X$ is connected, then $(X, p)$ is said to be a Riemann domain. We say that two Riemann regions $(X, p)$ and $(Y, q)$ over $\mathbb{C}^{n}$ are isomorphic (written $(X, p) \simeq(Y, q)$ ) if there exists a biholomorphic mapping $\varphi: X \rightarrow Y$ such that $q \circ \varphi=p$. Throughout, isomorphic Riemann regions will be identified.

We say that an open set $U \subset X$ is univalent (schlicht) if $\left.p\right|_{U}$ is injective. Note that $X$ is univalent $\operatorname{iff}(X, p) \simeq\left(\Omega, \operatorname{id}_{\Omega}\right)$, where $\Omega$ is an open set in $\mathbb{C}^{n}$.

Let $f \in \mathcal{O}(X)$. For any $\alpha \in \mathbb{Z}_{+}^{n}\left(\mathbb{Z}_{+}\right.$stands for the set of non-negative integers) and $x_{0} \in X$, let $D^{\alpha} f\left(x_{0}\right)$ denote the $\alpha$-partial derivative of $f$ at $x_{0}$,

$$
D^{\alpha} f\left(x_{0}\right):=D^{\alpha}\left(f \circ\left(\left.p\right|_{U}\right)^{-1}\right)\left(p\left(x_{0}\right)\right),
$$

where $U$ is an open univalent neighborhood of $x_{0}$ and $D^{\alpha}$ on the right hand side means the standard $\alpha$-partial derivative operator in $\mathbb{C}^{n}$. Let $T_{x_{0}} f$ denote the Taylor series of $f$ at $x_{0}$, i.e. the formal power series

$$
\sum_{\alpha \in \mathbb{Z}_{+}^{n}} \frac{1}{\alpha !} D^{\alpha} f\left(x_{0}\right)\left(z-p\left(x_{0}\right)\right)^{\alpha}, \quad z \in \mathbb{C}^{n} .
$$

For $x_{0} \in X$ and $0<r \leq \infty$ let $\mathbb{P}_{X}\left(x_{0}, r\right)$ denote an open univalent neighborhood of $x_{0}$ such that $p\left(\mathbb{P}_{X}\left(x_{0}, r\right)\right)=\mathbb{P}\left(p\left(x_{0}\right), r\right)=$ the polydisc

2000 Mathematics Subject Classification: 32D05, 32D25.

Key words and phrases: region of holomorphy, pluripolar set.

The research was supported by DFG grant no. 227/8-1,2 and Ministry of Scientific Research and Information Technology grant no. 1 PO3A 00528. 
with center at $p\left(x_{0}\right)$ and radius $r$. Let $d_{X}\left(x_{0}\right)$ denote the maximal $r$ such that $\mathbb{P}_{X}\left(x_{0}, r\right)$ exists. Put $\mathbb{P}_{X}\left(x_{0}\right):=\mathbb{P}_{X}\left(x_{0}, d_{X}\left(x_{0}\right)\right)$.

For $f \in \mathcal{O}(X)$ and $x_{0} \in X$, let $d\left(T_{x_{0}} f\right)$ denote the radius of convergence of $T_{x_{0}} f$, i.e.

$$
d\left(T_{x_{0}} f\right):=\sup \left\{r>0: \text { the series } T_{x_{0}} f \text { is convergent in } \mathbb{P}\left(p\left(x_{0}\right), r\right)\right\} .
$$

Obviously, $d\left(T_{x_{0}} f\right) \geq d_{X}\left(x_{0}\right)$ and $f(x)=T_{x_{0}} f(p(x)), x \in \mathbb{P}_{X}\left(x_{0}\right)$. Notice that

$$
\frac{1}{d\left(T_{x_{0}} f\right)}=\limsup _{\nu \rightarrow \infty}\left(\max _{\alpha \in \mathbb{Z}_{+}^{n}:|\alpha|=\nu} \frac{1}{\alpha !}\left|D^{\alpha} f\left(x_{0}\right)\right|\right)^{1 / \nu} .
$$

From now on we assume that all Riemann regions considered are countable at infinity.

Let $\emptyset \neq \mathcal{F} \subset \mathcal{O}(X)$. We say that $(X, p)$ is an $\mathcal{F}$-region of existence if

$$
d_{X}(x)=\inf \left\{d\left(T_{x} f\right): f \in \mathcal{F}\right\}, \quad x \in X .
$$

We say that an $\mathcal{F}$-region of existence $(X, p)$ is an $\mathcal{F}$-region of holomorphy if $\mathcal{F}$ weakly separates points in $X$, i.e. for any $x^{\prime}, x^{\prime \prime} \in X$ with $x^{\prime} \neq x^{\prime \prime}$ and $p\left(x^{\prime}\right)=p\left(x^{\prime \prime}\right)$, there exists an $f \in \mathcal{F}$ such that $T_{x^{\prime}} f \neq T_{x^{\prime \prime}} f$ (as formal power series).

REMARK 1.1 (Properties of regions of holomorphy). (a) Let $(X, p)$ be an $\mathcal{F}$-region of holomorphy and let $U \subset X$ be a univalent domain for which there exists a domain $V \supset p(U)$ such that for every $f \in \mathcal{F}$ there exists a function $F_{f} \in \mathcal{O}(V)$ such that $F_{f}=f \circ\left(\left.p\right|_{U}\right)^{-1}$ on $p(U)$. Then there exists a univalent domain $W \supset U$ with $p(W)=V$.

Indeed, we only need to observe that we may always assume that $(X, p)$ is realized as an open subset of the sheaf of $\mathcal{F}$-germs of holomorphic functions (cf. [Jar-Pfl 2000, proof of Theorem 1.8.4]) and, consequently, we may put

$$
W:=\left\{\left[\left(D,\left(F_{f}\right)_{f \in \mathcal{F}}\right)\right]_{z}: z \in V\right\}
$$

(cf. [Jar-Pfl 2000, Example 1.6.6]).

(b) ([Jar-Pfl 2000, Proposition 1.8.10]) Let $A \subset X$ be a dense subset such that $A=p^{-1}(p(A))$. Then the following conditions are equivalent:

(i) $(X, p)$ is an $\mathcal{F}$-region of holomorphy;

(ii) $d_{X}(x)=\inf \left\{d\left(T_{x} f\right): f \in \mathcal{F}\right\}, x \in A$, and for any $x^{\prime}, x^{\prime \prime} \in A$ with $x^{\prime} \neq x^{\prime \prime}$ and $p\left(x^{\prime}\right)=p\left(x^{\prime \prime}\right)$, there exists an $f \in \mathcal{F}$ such that $T_{x^{\prime}} f \neq T_{x^{\prime \prime}} f$.

(c) If $(X, p)$ is an $\mathcal{F}$-region of holomorphy, then there exists a finite or countable subfamily $\mathcal{F}_{0} \subset \mathcal{F}$ such that $(X, p)$ is an $\mathcal{F}_{0}$-region of holomorphy.

Indeed, we may assume that $X$ is connected. The case where $(X, p) \simeq$ $\left(\mathbb{C}^{n}, \mathrm{id}_{\mathbb{C}^{n}}\right)$ is trivial. Thus assume that $d_{X}(x)<\infty, x \in X$. Let $A \subset X$ be 
a countable dense subset such that $A=p^{-1}(p(A))$. By (b), for any $x \in A$ and $r>d_{X}(x)$ there exists an $f_{x, r} \in \mathcal{F}$ such that $d\left(T_{x} f_{x, r}\right)<r$, and for $x^{\prime}, x^{\prime \prime} \in A$ with $x^{\prime} \neq x^{\prime \prime}$ and $p\left(x^{\prime}\right)=p\left(x^{\prime \prime}\right)$, there exists an $f_{x^{\prime}, x^{\prime \prime}} \in \mathcal{F}$ such that $T_{x^{\prime}} f_{x^{\prime}, x^{\prime \prime}} \neq T_{x^{\prime \prime}} f_{x^{\prime}, x^{\prime \prime}}$. Now, we may take

$$
\begin{aligned}
\mathcal{F}_{0}:= & \left\{f_{x, r}: x \in A, \mathbb{Q} \ni r>d_{X}(x)\right\} \\
& \cup\left\{f_{x^{\prime}, x^{\prime \prime}}: x^{\prime}, x^{\prime \prime} \in A, x^{\prime} \neq x^{\prime \prime}, p\left(x^{\prime}\right)=p\left(x^{\prime \prime}\right)\right\} .
\end{aligned}
$$

2. Main results: separate holomorphy. Let $(X, p)$ be a Riemann domain over $\mathbb{C}^{n}=\mathbb{C}^{k} \times \mathbb{C}^{l}$,

$$
p=(u, v): X \rightarrow \mathbb{C}^{k} \times \mathbb{C}^{l} .
$$

Put $D:=p(X), D_{k}:=u(X), D^{l}:=v(X)$. For $a \in D_{k}$ define $X_{a}:=u^{-1}(a)$, $p_{a}:=\left.v\right|_{X_{a}}$. Similarly, for $b \in D^{l}$, put $X^{b}:=v^{-1}(b), p^{b}:=\left.u\right|_{X^{b}}$.

REMARK 2.1. For every $a \in D_{k},\left(X_{a}, p_{a}\right)$ is a Riemann region over $\mathbb{C}^{l}$, countable at infinity.

Let $\emptyset \neq \mathcal{F} \subset \mathcal{O}(X)$. For $a \in D_{k}$ define $f_{a}:=\left.f\right|_{X_{a}}, \mathcal{F}_{a}:=\left\{f_{a}: f \in \mathcal{F}\right\} \subset$ $\mathcal{O}\left(X_{a}\right)$, and analogously, $f^{b}:=\left.f\right|_{X^{b}}, \mathcal{F}^{b}:=\left\{f^{b}: f \in \mathcal{F}\right\} \subset \mathcal{O}\left(X^{b}\right), b \in D^{l}$.

The main result of the paper is the following

THEOREM 2.2.

(a) Let $\emptyset \neq \mathcal{F} \subset \mathcal{O}(X)$ and assume that $(X, p)$ is an $\mathcal{F}$-domain of holomorphy. Then there exists a pluripolar set $S_{k} \subset D_{k}$ such that for every $a \in D_{k} \backslash S_{k},\left(X_{a}, p_{a}\right)$ is an $\mathcal{F}_{a}$-region of holomorphy.

(b) Assume that $(X, p) \simeq\left(D, \mathrm{id}_{D}\right)$, where $D \subset \mathbb{C}^{k} \times \mathbb{C}^{l}$ is a fat domain (i.e. $D=\operatorname{int} \bar{D})$ and there exist sets $S_{k} \subset D_{k}, S^{l} \subset D^{l}$ such that:

- int $S_{k}=\emptyset$, int $S^{l}=\emptyset$,

- for any $a \in D_{k} \backslash S_{k}, D_{a}$ is an $\mathcal{F}_{a}$-region of holomorphy,

- for any $b \in D^{l} \backslash S^{l}, D^{b}$ is an $\mathcal{F}^{b}$-region of holomorphy.

Then $D$ is an $\mathcal{F}$-domain of holomorphy.

Proof. (a) By Remark 1.1(c), we may assume that $\mathcal{F}$ is finite or countable.

STEP 1. There exists a pluripolar set $P \subset D_{k}$ such that for any a $\in$ $D_{k} \backslash P,\left(X_{a}, p_{a}\right)$ is an $\mathcal{F}_{a}$-region of existence.

Define $R_{f, b}(x):=d\left(T_{x} f_{u(x)}\right), f \in \mathcal{F}, b \in D^{l}, x \in X^{b}$. Recall that

$$
\frac{1}{R_{f, b}(x)}=\limsup _{\nu \rightarrow \infty}\left(\max _{\beta \in \mathbb{Z}_{+}^{l}:|\beta|=\nu} \frac{1}{\beta !}\left|D^{(0, \beta)} f(x)\right|\right)^{1 / \nu}, \quad x \in X^{b} .
$$


Obviously, $R_{f, b}(x) \geq d_{X}(x), x \in X^{b}$. By the Cauchy inequalities, we get

$$
\begin{aligned}
& \frac{1}{\beta !}\left|D^{(0, \beta)} f(x)\right| \leq \frac{\sup _{\mathbb{P}_{X}\left(x_{0}, r\right)}|f|}{r^{|\beta|}}, \\
& \quad 0<r<d_{X}\left(x_{0}\right), x \in \mathbb{P}_{X}\left(x_{0}, r / 2\right), \beta \in \mathbb{Z}_{+}^{l} .
\end{aligned}
$$

Consequently, the function $-\log \left(R_{f, b}\right)_{*}($ where $*$ denotes the lower semicontinuous regularization on $\left.X^{b}\right)$ is plurisubharmonic on $X^{b}$. Put

$$
P_{f, b}:=u\left(\left\{x \in X^{b}:\left(R_{f, b}\right)_{*}(x)<R_{f, b}(x)\right\}\right) \subset D_{k} .
$$

It is known that $P_{f, b}$ is pluripolar (cf. [Jar-Pfl 2000, Theorem 2.1.41(b)]). Put

$$
R_{b}:=\inf _{f \in \mathcal{F}} R_{f, b}, \quad \widehat{R}_{b}:=\inf _{f \in \mathcal{F}}\left(R_{f, b}\right)_{*} .
$$

Observe that $-\log \left(\widehat{R}_{b}\right)_{*}$ is plurisubharmonic on $X^{b}$. Put

$$
P_{b}:=u\left(\left\{x \in X^{b}:\left(\widehat{R}_{b}\right)_{*}(x)<\widehat{R}_{b}(x)\right\}\right) \subset D_{k} .
$$

The set $P_{b}$ is also pluripolar (cf. [Jar-Pfl 2000, Theorem 2.1.41(a)]). Now let $B \subset D^{l}$ be a dense countable set. Define

$$
P:=\left(\bigcup_{f \in \mathcal{F}, b \in B} P_{f, b}\right) \cup\left(\bigcup_{b \in B} P_{b}\right) \subset D_{k} .
$$

Then $P$ is pluripolar.

Take an $a \in D_{k} \backslash P$ and suppose that $X_{a}$ is not an $\mathcal{F}_{a}$-region of existence. Then there exist a point $x_{0} \in X_{a}$ and a number $r>d_{X_{a}}\left(x_{0}\right)$ such that $b:=v\left(x_{0}\right) \in B$ and $R_{b}\left(x_{0}\right)>r$. Since $a \notin P$, we have

$$
\left(\widehat{R}_{b}\right)_{*}\left(x_{0}\right)=\widehat{R}_{b}\left(x_{0}\right)=\inf _{f \in \mathcal{F}}\left(R_{f, b}\right)_{*}=\inf _{f \in \mathcal{F}} R_{f, b}=R_{b}\left(x_{0}\right)>r .
$$

In particular, there exists $0<\varepsilon<d_{X}\left(x_{0}\right)$ such that $\left(\widehat{R}_{b}\right)_{*}(x)>r$ for $x \in \mathbb{P}_{X^{b}}\left(x_{0}\right)$. Since

$$
R_{b}(x)=\inf _{f \in \mathcal{F}} R_{f, b}(x) \geq \inf _{f \in \mathcal{F}}\left(R_{f, b}\right)_{*}(x)=\widehat{R}_{b}(x) \geq\left(\widehat{R}_{b}\right)_{*}(x),
$$

we conclude that $R_{b}(x)>r$ for $x \in \mathbb{P}_{X^{b}}\left(x_{0}\right)$. Put $U:=\mathbb{P}_{X}\left(x_{0}, \varepsilon\right)$. Hence, by the classical Hartogs lemma, for every $f \in \mathcal{F}$, the function $f \circ\left(\left.p\right|_{U}\right)^{-1}$ extends holomorphically to $V:=\mathbb{P}(a, \varepsilon) \times \mathbb{P}(b, r)$. Since $(X, p)$ is an $\mathcal{F}$-domain of holomorphy, by Remark 1.1(a), there exists a univalent domain $W \subset X$, $U \subset W$, such that $p(W)=V$. In particular, $d_{X_{a}}\left(x_{0}\right) \geq r$, a contradiction.

STEP 2. There exists a pluripolar set $P \subset D_{k}$ such that for any $a \in$ $D_{k} \backslash P$ the family $\mathcal{F}_{a}$ weakly separates points in $X_{a}$.

Take $a \in D_{k}, x^{\prime}, x^{\prime \prime} \in X_{a}$ with $x^{\prime} \neq x^{\prime \prime}$ and $p_{a}\left(x^{\prime}\right)=p_{a}\left(x^{\prime \prime}\right)=: b$. Since $\mathcal{F}$ weakly separates points in $X$, there exists an $f \in \mathcal{F}$ such that $T_{x^{\prime}} f \neq T_{x^{\prime \prime}} f$. 
Put $r:=\min \left\{d\left(T_{x^{\prime}} f\right), d\left(T_{x^{\prime \prime}} f\right)\right\}$ and let

$$
P_{a, x^{\prime}, x^{\prime \prime}}:=\bigcap_{w \in \mathbb{P}(b, r)}\left\{z \in \mathbb{P}(a, r): T_{x^{\prime}} f(z, w)=T_{x^{\prime \prime}} f(z, w)\right\} .
$$

Then $P_{a, x^{\prime}, x^{\prime \prime}} \varsubsetneqq \mathbb{P}(a, r)$ is an analytic subset. For any $z \in \mathbb{P}(a, r) \backslash P_{a, x^{\prime}, x^{\prime \prime}}$ we have $T_{x^{\prime}} f(z, \cdot) \not \equiv T_{x^{\prime \prime}} f(z, \cdot)$ on $\mathbb{P}(b, r)$.

Take a countable dense set $A \subset D_{k}$. For any $a \in A$ let $B_{a} \subset X_{a}$ be a countable dense subset such that $p_{a}^{-1}\left(p_{a}\left(B_{a}\right)\right)=B_{a}$. Then

$$
P:=\bigcup_{\substack{a \in A, x^{\prime}, x^{\prime \prime} \in B_{a} \\ x^{\prime} \neq x^{\prime \prime}, p_{a}\left(x^{\prime}\right)=p_{a}\left(x^{\prime \prime}\right)}} P_{a, x^{\prime}, x^{\prime \prime}}
$$

is a pluripolar set.

Fix $a_{0} \in D_{k} \backslash P, x_{0}^{\prime}, x_{0}^{\prime \prime} \in X_{a_{0}}$ with $x_{0}^{\prime} \neq x_{0}^{\prime \prime}$ and $p_{a_{0}}\left(x_{0}^{\prime}\right)=p_{a_{0}}\left(x_{0}^{\prime \prime}\right)=: b_{0}$. Put $r:=\min \left\{d_{X}\left(x_{0}^{\prime}\right), d_{X}\left(x_{0}^{\prime \prime}\right)\right\}$. Let $a \in A \cap \mathbb{P}\left(a_{0}, r / 2\right)$ and $x^{\prime}, x^{\prime \prime} \in B_{a}$ be such that $x^{\prime} \in \mathbb{P}_{X}\left(x_{0}^{\prime}, r / 2\right), x^{\prime \prime} \in \mathbb{P}_{X}\left(x_{0}^{\prime \prime}, r / 2\right)$ and $p_{a}\left(x^{\prime}\right)=p_{a}\left(x^{\prime \prime}\right)$. Since $a_{0} \notin P$, we conclude that $T_{x^{\prime}} f\left(a_{0}, \cdot\right) \not \equiv T_{x^{\prime \prime}} f\left(a_{0}, \cdot\right)$ on $\mathbb{P}\left(b_{0}, r / 2\right)$. Consequently, $T_{x_{0}^{\prime}} f\left(a_{0}, \cdot\right) \not \equiv T_{x_{0}^{\prime \prime}} f\left(a_{0}, \cdot\right)$ on $\mathbb{P}\left(b_{0}, r / 2\right)$, which implies that $T_{x_{0}^{\prime}} f_{a_{0}}$ $\neq T_{x_{0}^{\prime \prime}} f_{a_{0}}$.

(b) Suppose there exist $x_{0}=\left(a_{0}, b_{0}\right) \in G$ and $r>d_{D}\left(x_{0}\right)=: r_{0}$ such that $d\left(T_{x_{0}} f\right) \geq r$ for $f \in \mathcal{F}$. Then $d\left(T_{b_{0}} f_{a}\right) \geq r$ for any $f \in \mathcal{F}$ and $a \in \mathbb{P}\left(a_{0}, r_{0}\right)$. Consequently, $\left(\mathbb{P}\left(a_{0}, r_{0}\right) \backslash S_{k}\right) \times \mathbb{P}\left(b_{0}, r\right) \subset D$. Since int $S_{k}=\emptyset$ and $D$ is fat, we conclude that $\mathbb{P}\left(a_{0}, r_{0}\right) \times \mathbb{P}\left(b_{0}, r\right) \subset D$. Now, we see that $d\left(T_{a_{0}} f^{b}\right) \geq r$ for any $f \in \mathcal{F}$ and $b \in \mathbb{P}\left(b_{0}, r\right)$. Consequently, $\mathbb{P}\left(a_{0}, r\right) \times\left(\mathbb{P}\left(b_{0}, r\right) \backslash S^{l}\right) \subset D$. Hence $\mathbb{P}\left(a_{0}, r\right) \times \mathbb{P}\left(b_{0}, r\right) \subset D$, a contradiction.

REMARK 2.3. The following natural question arises from the discussion above: is it possible to sharpen Theorem 2.2(a) so that the exceptional set there is even a countable union of locally analytic sets? The following example will show that the answer is, in general, negative.

Let $C_{1} \subset \mathbb{D}$ (the unit disc) be a compact polar set which is uncountable (take, for example, an appropriate Cantor set). Define $C:=C_{1} \cup C_{2}$, where $C_{2}:=\mathbb{D} \cap \mathbb{Q}^{2}$. Then $C$ is polar and a countable union of compact sets.

Using Example 2 from [Ter 1972], we find a function $f: \mathbb{D} \times \mathbb{D} \rightarrow \mathbb{C}$ with the following properties:

- $f(\cdot, w) \in \mathcal{O}(\mathbb{D})$ for all $w \in \mathbb{D}$,

- $f(z, \cdot) \in \mathcal{O}(\mathbb{D})$ for all $z \in C$,

- $f$ is unbounded near some point $\left(z_{0}, 0\right) \in \mathbb{D} \times \mathbb{D}$.

Using the corollary to Lemma 8 of [Ter 1972], we conclude that there is a non-empty domain $V \subset \subset \mathbb{D}$ such that $\left.f\right|_{\mathbb{D} \times V} \in \mathcal{O}(\mathbb{D} \times V)$. Set $\mathcal{F}:=$ $\left\{\left.f\right|_{\mathbb{D} \times V},\left.g\right|_{\mathbb{D} \times V}\right\}$, where $g \in \mathcal{O}(\mathbb{D} \times \mathbb{D})$ is chosen in such a way that $\mathbb{D} \times \mathbb{D}$ is the existence domain of $g$. Denote by $\left(D^{\prime}, p\right)$ the $\mathcal{F}$-envelope of holomorphy 
of $\mathbb{D} \times V$. Then $p\left(D^{\prime}\right) \subset \mathbb{D} \times \mathbb{D}$. Moreover, using the fact that $C$ is dense in $\mathbb{D}$ one sees that $D^{\prime}$ is univalent. Indeed, let us take a sequence $G_{j}=G_{j}^{\prime} \times G_{j}^{\prime \prime} \subset$ $\mathbb{D} \times \mathbb{D}, j=1, \ldots, N$, of bidiscs, $G_{j} \cap G_{j+1} \neq \emptyset$, and functions $f_{j} \in \mathcal{O}\left(G_{j}\right), j=$ $1, \ldots, N$, such that $G_{1} \subset \mathbb{D} \times V, f_{1}=\left.f\right|_{G_{1}}$, and $\left.f_{j}\right|_{G_{j} \cap G_{j+1}}=\left.f_{j+1}\right|_{G_{j} \cap G_{j+1}}$, $j=1, \ldots, N-1$. We claim that then $f_{N}=\left.f\right|_{G_{N}}$, which implies that $D^{\prime}$ is univalent. By induction we may assume that $f_{j}=\left.f\right|_{G_{j}}$ for a $j<N$. Then for any point $a \in C \cap G_{j}^{\prime} \cap G_{j+1}^{\prime}$ we have two holomorphic functions $f(a, \cdot)$ and $f_{j+1}(a, \cdot)$ on $G_{j+1}^{\prime \prime}$. They coincide on $G_{j}^{\prime \prime} \cap G_{j+1}^{\prime \prime}$, and so on $G_{j+1}^{\prime \prime}$. Now fix a $b \in G_{j+1}^{\prime \prime}$. Then $f(\cdot, b)$ and $f_{j+1}(\cdot, b)$ are holomorphic in $G_{j+1}^{\prime}$ and they coincide on $C \cap G_{j}^{\prime} \cap G_{j+1}^{\prime}$; hence they are equal on $G_{j+1}^{\prime \prime}$, i.e. $\left.f\right|_{G_{j+1}}=f_{j+1}$.

Set $D:=p\left(D^{\prime}\right)$. Then $D$ is an $\widehat{\mathcal{F}}$-domain of holomorphy, where

$$
\widehat{\mathcal{F}}:=\left\{\widehat{g}:=\left.g\right|_{D}, \widehat{f}:=\left.f\right|_{D}\right\} .
$$

Observe that for any $a \in C$, the functions $\widehat{f}(a, \cdot), \widehat{g}(a, \cdot)$ extend to the whole of $\mathbb{D}$.

Fix $R^{\prime}<R \in(0,1)$ such that $V \subset \subset \mathbb{P}\left(0, R^{\prime}\right)$. Suppose that there is an $a_{0} \in C$ with $\left\{a_{0}\right\} \times \mathbb{P}(0, R) \subset D$. Then there is a small open neighborhood $U \subset \mathbb{D}$ of $a_{0}$ such that $U \times \mathbb{P}\left(0, R^{\prime}\right) \subset D$. In view of the Hartogs lemma we conclude that $f$ is holomorphic on $\mathbb{D} \times \mathbb{P}\left(0, R^{\prime}\right)$, in particular a holomorphic extension of $\left.f\right|_{\mathbb{D} \times V}$, and therefore bounded near $\left(z_{0}, 0\right)$, a contradiction. Thus, the singular set $S_{1}$ for $D$ must contain $C$.

REMARK 2.4. Observe that Theorem 2.2(b) need not be true if $D$ is not fat. For example, let $D:=\mathbb{D}^{2} \backslash\{(0,0)\} \subset \mathbb{C}^{2}, \mathcal{F}:=\mathcal{O}(D)$. By the Hartogs extension theorem, any function from $\mathcal{F}$ extends holomorphically to $\mathbb{D}^{2}$. Thus $D$ is not an $\mathcal{F}$-domain of holomorphy. Observe that for any $a \in \mathbb{D} \backslash\{0\}, D_{a}=\mathbb{D}$ and $\mathcal{F}_{a}=\mathcal{O}(\mathbb{D})$. Similarly, for any $b \in \mathbb{D} \backslash\{0\}, D^{b}=\mathbb{D}$ and $\mathcal{F}^{b}=\mathcal{O}(\mathbb{D})$.

3. Applications: separately holomorphic functions. Directly from Theorem 2.2 we get the following useful corollary.

Corollary 3.1. Let $D \subset \mathbb{C}^{k} \times \mathbb{C}^{l}$ be a domain, let $\emptyset \neq \mathcal{F} \subset \mathcal{O}(D)$ and let $A \subset \operatorname{pr}_{\mathbb{C}^{k}}(D)$. Assume that for any $a \in A$ we are given a domain $G(a) \supset D_{a}$ in $\mathbb{C}^{l}$ such that:

- for any $f \in \mathcal{F}$, the function $f(a, \cdot)$ extends to an $\widehat{f}_{a} \in \mathcal{O}(G(a))$,

- the domain $G(a)$ is an $\left\{\widehat{f}_{a}: f \in \mathcal{F}\right\}$-domain of holomorphy.

Let $(X, p)$ be the $\mathcal{F}$-envelope of holomorphy of $D$. Then there exists a pluripolar set $P \subset A$ such that for every $a \in A \backslash P$ we have $\left(X_{a}, p_{a}\right) \simeq\left(G(a), \operatorname{id}_{G(a)}\right)$.

Recall a version of the cross theorem for separately holomorphic functions with pluripolar singularities (cf. [Jar-Pfl 2003, Main Theorem]). 
Theorem 3.2. Let $D \subset \mathbb{C}^{k}, G \subset \mathbb{C}^{l}$ be domains of holomorphy and let $A \subset D, B \subset G$ be locally pluriregular sets $\left({ }^{1}\right)$. Consider the cross

$$
X=\mathbb{X}(A, B ; D, G):=(A \times G) \cap(D \times B)
$$

and let

$$
\widehat{X}=\widehat{\mathbb{X}}(A, B ; D, G):=\left\{(z, w) \in D \times G: \omega_{A, D}(z)+\omega_{B, G}(w)<1\right\},
$$

where $\omega_{A, D}$ and $\omega_{B, G}$ are generalized relative extremal functions. Let $M \subset X$ be a relatively closed set $\left({ }^{2}\right)$ such that:

- for every $a \in A$ the fiber $M_{a}:=\{w \in G:(a, w) \in M\}$ is pluripolar,

- for every $b \in B$ the fiber $M^{b}:=\{z \in D:(z, b) \in M\}$ is pluripolar.

Let $\mathcal{F}=\mathcal{O}_{\mathrm{s}}(X \backslash M)$ denote the set of all functions separately holomorphic on $X \backslash M$, i.e. of those functions $f: X \backslash M \rightarrow \mathbb{C}$ for which:

- for every $a \in A, f(a, \cdot) \in \mathcal{O}\left(G \backslash M_{a}\right)$,

- for every $b \in A, f(\cdot, b) \in \mathcal{O}\left(D \backslash M^{b}\right)$.

Then there exists a relatively closed pluripolar set $S \subset \widehat{X}$ such that:

- $S \cap X \subset M$,

- for every $f \in \mathcal{F}$ there exists an $\widehat{f} \in \mathcal{O}(\widehat{X} \backslash S)$ with $\widehat{f}=f$ on $X \backslash M$,

- $\widehat{X} \backslash S$ is an $\{\widehat{f}: f \in \mathcal{F}\}$-domain of holomorphy.

In the proof presented in [Jar-Pfl 2003] the assumption that $M$ is relatively closed in $X$ played an important role. Observe that from the point of view of the formulation of the above theorem, we only need to assume that all the fibers $M_{a}$ and $M^{b}$ are relatively closed. Corollary 3.1 permits us to clarify this problem in certain cases.

Lemma 3.3. Let $D \subset \mathbb{C}^{k}, G_{0} \subset G \subset \mathbb{C}^{l}$ be domains of holomorphy and let $A \subset D$. Assume that for every $a \in A$ we are given a relatively closed pluripolar set $M(a) \subset G$. Let $\mathcal{F}$ denote the set of all functions $f \in$ $\mathcal{O}\left(D \times G_{0}\right)$ such that for every $a \in A$, the function $f(a, \cdot)$ extends to an $\widehat{f}_{a} \in \mathcal{O}(G \backslash M(a))$. Assume that for every $a \in A$ the set $M(a)$ is singular with respect to the family $\left\{\widehat{f}_{a}: f \in \mathcal{F}\right\}\left({ }^{3}\right)$. Then there exists a pluripolar

$\left({ }^{1}\right)$ A non-empty set $A$ is said to be locally pluriregular if for any $a \in A$, the set $A$ is locally pluriregular at $a$, i.e. for any open neighborhood $U$ of $a$ we have $h_{A \cap U, U}^{*}(a)=0$, where $h_{A \cap U, U}$ denotes the relative extremal function of $A \cap U$ in $U$. For an arbitrary set $A$ define $A^{*}:=\{a \in \bar{A}: A$ is locally pluriregular at $a\}$. It is known that the set $Z:=A \backslash A^{*}$ is pluripolar. In particular, if $A$ is non-pluripolar, then $A \backslash Z$ is locally pluriregular.

$\left({ }^{2}\right)$ That is, $M$ is closed in $X$.

$\left({ }^{3}\right)$ Recall that a relatively closed pluripolar set $M \subset G$ is said to be singular with respect to a family $\emptyset \neq \mathcal{G} \subset \mathcal{O}(G \backslash M)$ if there is no point $a \in M$ which has an open neighborhood $U \subset G$ such that every function from $\mathcal{G}$ extends holomorphically to $U$ (cf. [Jar-Pfl 2000, §3.4]). 
set $P \subset A$ such that if we put $A_{0}:=A \backslash P$, then the set

$$
M\left(A_{0}\right):=\bigcup_{a \in A_{0}}\{a\} \times M(a)
$$

is relatively closed in $A_{0} \times G$.

Proof. First observe that every function from $\mathcal{O}(G)$ may be regarded as an element of $\mathcal{F}$, which implies that for every $a \in A$ the domain $G(a):=$ $G \backslash M(a)$ is a $\left\{\widehat{f}_{a}: f \in \mathcal{F}\right\}$-domain of holomorphy.

Let $(X, p)$ be the $\mathcal{F}$-envelope of holomorphy of $D \times G_{0}$. Since $D$ and $G$ are domains of holomorphy, we may assume that $p(X) \subset D \times G$.

By Corollary 3.1, there exists a pluripolar set $P \subset A$ such that for every $a \in A_{0}:=A \backslash P$ we have $\left(X_{a}, p_{a}\right) \simeq\left(G(a), \operatorname{id}_{G(a)}\right)$. Thus $p$ is injective on the set $B:=p^{-1}\left(A_{0} \times G\right)$ and $p(B)=\bigcup_{a \in A_{0}}\{a\} \times G(a)=\left(A_{0} \times G\right) \backslash M\left(A_{0}\right)$. Hence $p(B)=p(X) \cap\left(A_{0} \times G\right)$ and, consequently, $p(B)$ is relatively open in $A_{0} \times G$.

Consequently, we get the following generalization of Theorem 3.2.

Theorem 3.4. Let $D_{0} \subset D \subset \mathbb{C}^{k}, G_{0} \subset G \subset \mathbb{C}^{l}$ be domains of holomorphy and let $A \subset D_{0}, B \subset G_{0}$ be non-pluripolar sets. Let $M \subset X:=$ $\mathbb{X}(A, B ; D, G)$ be such that:

- for every $a \in A$ the fiber $M_{a}$ is a closed pluripolar subset of $G$,

- for every $b \in B$ the fiber $M^{b}$ is a closed pluripolar subset of $D$.

Let $\mathcal{F}$ denote the set of all functions $f \in \mathcal{O}\left(D_{0} \times G_{0}\right)$ such that:

- for every $a \in A$ the function $f(a, \cdot)$ extends holomorphically to $G \backslash M_{a}$,

- for every $b \in A$ the function $f(\cdot, b)$ extends holomorphically to $D \backslash M^{b}$.

Then there exist:

- pluripolar sets $P \subset A, Q \subset B$ such that the sets $A_{0}:=A \backslash P$ and $B_{0}:=B \backslash Q$ are locally pluriregular,

- a relatively closed pluripolar set $S \subset \widehat{X}_{0}:=\widehat{\mathbb{X}}\left(A_{0}, B_{0} ; D, G\right)$

such that:

- $S \cap \mathbb{X}\left(A_{0}, B_{0} ; D, G\right) \subset M$,

- for every $f \in \mathcal{F}$ there exists an $\widehat{f} \in \mathcal{O}\left(\widehat{X}_{0} \backslash S\right)$ with $\widehat{f}=f$ on $D_{0} \times G_{0}$,

- $\widehat{X}_{0} \backslash S$ is an $\{\widehat{f}: f \in \mathcal{F}\}$-domain of holomorphy.

REMARK 3.5. More general versions of the cross theorem (also for $N$-fold crosses) will be discussed in our forthcoming paper.

Acknowledgments. The authors wish to thank Professor W. Zwonek for helpful remarks. 


\section{References}

[Jar-Pfl 2000] M. Jarnicki and P. Pflug, Extension of Holomorphic Functions, de Gruyter Exp. Math. 34, de Gruyter, 2000.

[Jar-Pfl 2003] —, -, An extension theorem for separately holomorphic functions with pluripolar singularities, Trans. Amer. Math. Soc. 355 (2003), 1251-1267.

[Ter 1972] T. Terada, Analyticités relatives à chaque variable, J. Math. Kyoto Univ. 12 (1972), 263-296.

Institute of Mathematics

Jagiellonian University

Reymonta 4

30-059 Kraków, Poland

E-mail: Marek.Jarnicki@im.uj.edu.pl
Institut für Mathematik Carl von Ossietzky Universität Oldenburg Postfach 2503

D-26111 Oldenburg, Germany E-mail: pflug@mathematik.uni-oldenburg.de

Received July 18, 2005

Revised version March 1, 2006 